\title{
Nano hemostat solution: immediate hemostasis at the nanoscale
}

\begin{abstract}
Hemostasis is a major problem in surgical procedures and after major trauma. There are few effective methods to stop bleeding without causing secondary damage. We used a self-assembling peptide that establishes a nanofiber barrier to achieve complete hemostasis in less than 15 seconds when applied directly to a wound in the brain, spinal cord, femoral artery, liver, or skin of mammals. This novel therapy stops bleeding without the use of pressure, cauterization, vasoconstriction, coagulation, or cross-linked adhesives. The self-assembling solution is nontoxic and nonimmunogenic, and the breakdown products are amino acids, which are tissue building blocks that can be used to repair the site of injury. Here we report the first use of nanotechnology to achieve complete hemostasis in less than 15 seconds, which could fundamentally change how much blood is needed during surgery of the future. (C) 2006 Published by Elsevier Inc.
\end{abstract}

Key words: $\quad$ Hemostasis; Surgery; Trauma; Nanotechnology; Self-assembling peptide

Through the ages doctors have found ways to achieve hemostasis, beginning with the simple act of applying pressure, then cauterization, ligation, and clinically induced vasoconstriction [1-10], but nanotechnology brings new possibilities for changes in medical technology. Here we present a novel method to stop bleeding using materials that self-assemble at the nanoscale when applied to a wound. This

The authors declare a competing financial interest: S.Z. is a co-founder and board member of 3D Matrix, the licensor of one of the materials used.

This work was supported by grants from the Deshpande Center for Technological Innovation at the Massachusetts Institute of Technology and the Research Grant Council (RGC) of Hong Kong/ and the NSF

[R.G.E.-B., D.K.C.T., G.E.S., and K-F.S. wrote and edited the paper; KF.S. and D.K.C.T. provided financial support; R.G.E.-B., Y-X.L., P.W.F.K., and W.W. performed surgery; R.G.E.-B., K-F.S., D.K.C.T., Y-X.L., P.W.F.K., and G.E.S. analyzed data; S.Z. provided material].

* Corresponding author. Department of Brain and Cognitive Sciences, 46-6007, Massachusetts Institute of Technology, Cambridge, MA 02139, USA.

*

E-mail address: rutledg@mit.edu (R.G. Ellis-Behnke). method results in the formation of a nanofiber barrier that 34 stops bleeding in any wet ionic environment in the body; 35 furthermore, the material is broken down into natural l-amino 36 acids that can be used by the surrounding tissue for repair. 37

Currently there are three basic categories of hemostatic 38 agents or procedures: chemical, thermal, and mechanical 39 [1,3,6,8,10-15]. Chemical agents are those that change 40 the clotting activity of the blood or act as vasoconstrictors, 41 such as thromboxane A2 [16], which causes vessels to 42 contract thus reducing blood flow and promoting clotting 43 $[7,16,17]$. Thermal devices commonly involve cauterization 44 using electrodes, lasers $[8,14]$, or heat. There are also agents 45 that react exothermically upon application that may 46 create an effect similar to a standard two probe cautery 47 device $[1,14]$. Mechanical methods use pressure or ligature 48 to slow the blood flow [3]. A combination therapy might use 49 both chemical and mechanical means to produce a he- 50 mostat that adsorbs fluid and swells [18], producing 51 pressure to slow the blood flow and allow clotting, or it 52 may involve the introduction of fibrinogen, thrombin, and 53 
A

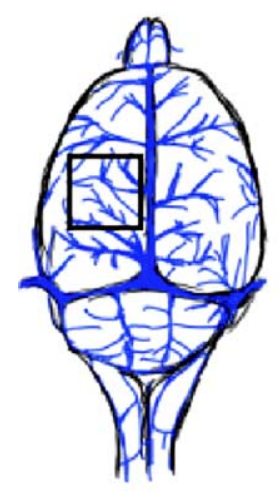

B

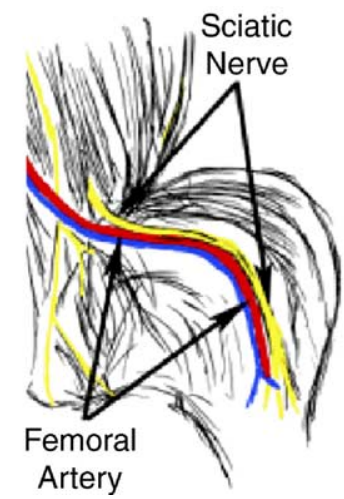

C

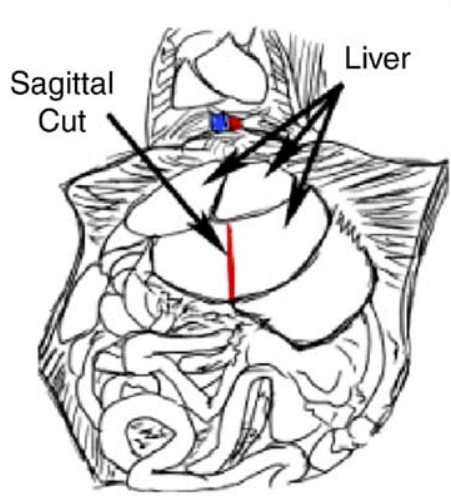

D

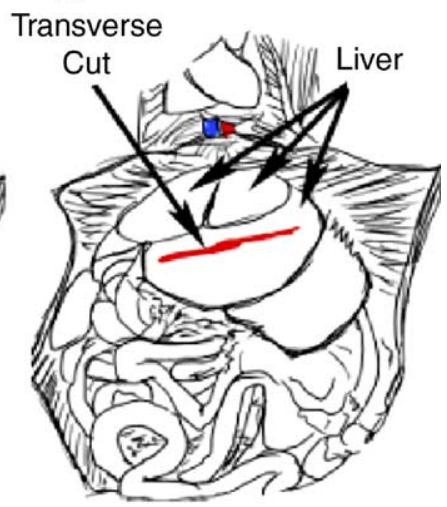

Fig 1. Schematics of surgical procedures. Rostral is up and caudal is down in all figures. A, Dorsal view of the rat brain. The blue lines depict the blood vessels superficial to the cortex. The boxed area corresponds to location of the lesion and treatment. B. Drawing of ventral view of the lower limb of a rat with the femoral artery in red and sciatic nerve in yellow. $\mathbf{C}$ and $\mathbf{D}$, Drawings of a ventral view of rat with abdomen open. Overlying structures have been removed exposing the liver. The lobe was transected with a cut (depicted in red) in both sagittal (C) and transverse (D) directions.

calcium to produce fibrin glue, which acts as an artificial clot $[1,2,5,6,8,10,14,19]$.

There are five major issues related to the limitations and applicability of many of these hemostatic agents. First, some of the materials are solid, such as powder formulations, and are not able to flow into the area of injury to bring about their hemostatic effects $[1,10,14]$; second, some liquid agents, such as cyanoacrylates, require a dry environment to be effective [8]; third, some materials can create an immune response resulting in the death of adjacent cells, placing additional stress on the body that can prolong or prevent healing $[8,10,14,15,20]$; fourth, some agents have a short shelf-life and very specific handling requirements $[6,10,14,16,17]$; and finally, many currently used hemostats are difficult to use in uncontrolled environments $[1,7,8,10,14]$. Moreover, if a therapy uses swelling as part of its hemostatic action, then extra care must be taken to ensure that the local blood supply is not reduced or stopped, which could cause additional tissue damage or even death. This is particularly crucial when using expanding foams [19]. Many hemostatic agents must be prepared just before use because of their short shelf-life. Surgical instruments, such as cauterization devices, clamps and clips, must be used by a skilled individual in a controlled environment $[2,5,8-10,16,20]$.

Our discovery, observed during a neurosurgical procedure, introduces a new way to stop bleeding using a selfassembling peptide that establishes a nanofiber barrier and incorporates it into the surrounding tissue to form an extracellular matrix (ECM). Surmising that nanotechnology might be useful in our central nervous system regeneration studies, we injected the material into wound sites in the brain of hamsters to determine whether it would facilitate neuronal regeneration [21]. To our surprise, it also stopped bleeding.

We then wanted to know if the rapid hemostasis that we had observed in our nerve regeneration experiments was tissue specific or would also work in other tissues. The seven experiments we designed and performed demonstrate that in 90 less than 15 seconds complete hemostasis can be achieved 91 after (1) a transection of a blood vessel leading to the superior 92 sagittal sinus in both hamsters and rats, (2) a spinal cord cut, 93 (3) a femoral artery cut, (4) a sagittal transection of the left 94 lateral liver lobe, (5) a transverse transection of the left lateral 95 liver lobe including a cut in a primary branch of the portal 96 vein, (6) a 4-mm liver punch biopsy, and (7) multiple 4-mm 97 skin punch biopsies on nude mice.

\section{Materials and methods}

Adult Syrian hamsters were anesthetized with an intra- 100 peritoneal injection of sodium pentobarbital $(50 \mathrm{mg} / \mathrm{kg})$, and 101 adult rats were anesthetized with an intraperitoneal injection 102 of ketamine $(50 \mathrm{mg} / \mathrm{kg})$. The experimental procedures 103 adhered strictly to the protocol approved by the Department 104 of Health and endorsed by the Committee on the Use of 105 Laboratory Animals for Teaching and Research of the 106 University of Hong Kong and the Massachusetts Institute 107 of Technology Committee on Animal Care.

\section{Cortical vessel cut experiment}

The animals were fitted in a head holder. The left lateral 110 part of the cortex was exposed, and each animal received a 111 transection of a blood vessel leading to the superior sagittal 112 sinus (Figure 1, A). With the aid of a sterile glass micro- 113 pipette, $20 \mu \mathrm{L}$ of $1 \%$ NHS-1 solution (see below under 114Q3 "Preparation of the self-assembling solutions") was applied to 115 the site of injury or iced saline in the control cases. The 116 animals were allowed to survive for as long as 6 months.

\section{Spinal cord injury experiment}

Under an operating microscope, the second thoracic 119 spinal cord segment (T2) was identified before performing a 120 

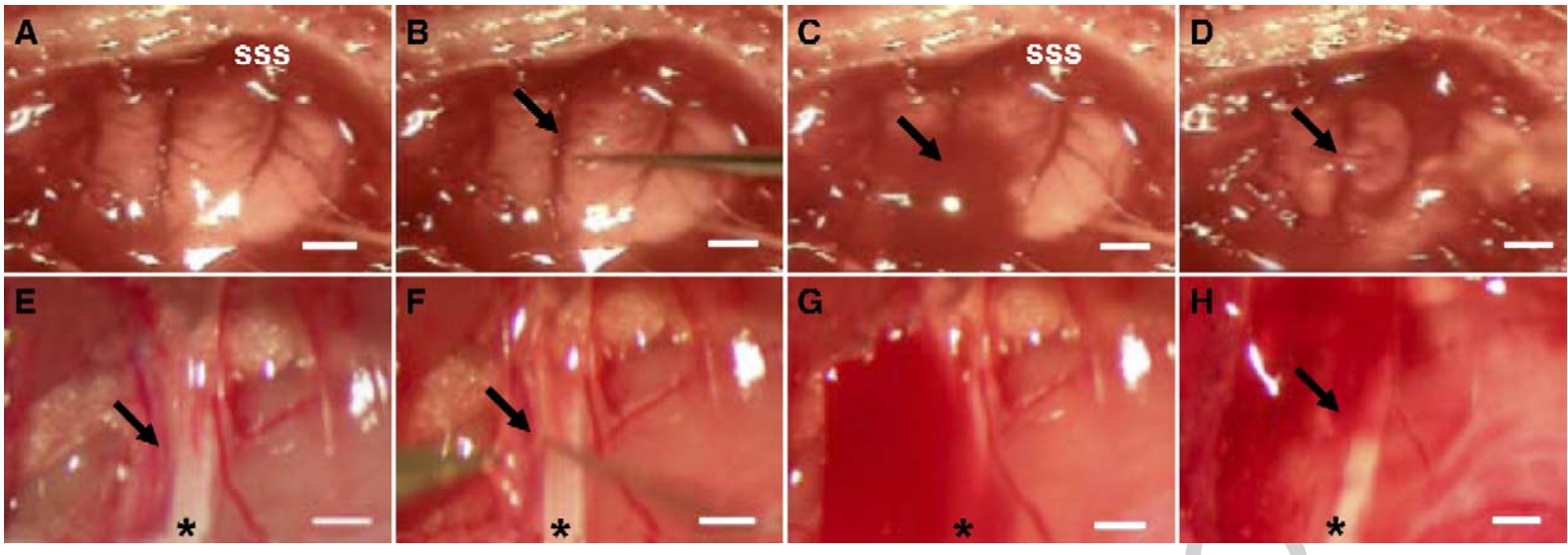

Fig 2. Complete hemostasis in brain and femoral artery. The pictures are time-lapse images at each stage of the experiment for brain (A - D) and femoral artery (E-H). A-D, Adult rat cortex hemostasis. Part of the overlying skull has been removed in an adult rat, and one of the veins of the superior sagittal sinus is transected and treated with $1 \%$ self-assembling NHS-1. A, The brain and veins of the superior sagittal sinus (SSS) are exposed. B, Cutting of the vein (arrow). C, Bleeding of the ruptured vein (arrow). D, The same area 5 seconds after application of the self-assembling NHS-1 to the location of the cut (arrow) as seen under the clear NHS-1. E-H, Rat femoral artery hemostasis. Exposure of the neurovascular bundle in the thigh showing the sciatic nerve $(*)$ in each panel. E, Femoral artery and vein exposed. F, Cutting of the artery (arrow). G, Bleeding, masking the artery completely and sciatic nerve partially. H, The same area 5 seconds after application of the self-assembling peptide to the cut (arrow). Note that there is complete hemostasis in the area formed by NHS-1 (covering the entire picture) as it self-assembles in the presence of blood and plasma, revealing the underlying structures. Complete hemostasis was achieved in $10.6 \pm 4.1$ seconds, significantly different from $367.5 \pm 37.7$ seconds in controls irrigated with saline $(P>.0001)$. Scale bars represent $1 \mathrm{~mm}$.

121 dorsal laminectomy in anesthetized adult rats [22,23]. After

122 opening the dura mater, we performed a right hemisection

123 using a ceramic knife. Immediately after the cord hemiQ3124 section $20 \mu \mathrm{L}$ of a $1 \%$ solution of NHS-1 was applied to the

125 area of the cut for bleeding control. The controls received a

126 saline treatment. The animals were allowed to survive for as

127 long as 8 weeks as part of another experiment.

\section{Femoral artery cut experiment}

129 Rats were placed on their backs, and the hind limb was 130 extended to expose the medial aspect of the thigh (Figure 1,

$131 \mathrm{~B}$ ). The skin was removed, and the overlying muscles were

132 cut to expose the femoral artery and sciatic nerve. The

133 femoral artery was cut to produce a high-pressure bleeder Q3134 (Figure 2, F). With a 27-gauge needle, $200 \mu \mathrm{L}$ of $1 \%$ NHS-

1351 solution was applied over the site of injury. In two cases Q4136 we applied the dry powder of NHS-1 to the injury site, 137 which also was effective. (Data are not shown and were not 138 included in the analysis.) Controls were treated with a 139 combination of saline and pressure with a gauge. All 140 animals were killed 4 hours after the experiment.

\section{Liver wound experiments}

142 Rats were anesthetized and placed on their back, and 143 the abdomen was opened exposing the liver (Figure 1, C). 144 The left lobe of the liver was cut using a scalpel in the 145 rostral-to-caudal direction, separating the two halves of the 146 lobe (Figure 3,B) in the sagittal cut. With a 27-gauge Q5 147 needle, $100 \mu \mathrm{L}$ of $1 \%$ or $2 \%$ NHS-1, NHS-2, or TM-3 148 solution was applied to the site of injury (Figure 3, B). 149 Livers of the controls were treated with saline or 150 cauterized. Cauterization was performed using a thermal cautery device and was applied to the entire surface of the 151 injury. In another group of 28 adult rats the same 152 procedure was followed for the liver, which was cut 153 transversely (Figure 3,D). With a 27-gauge needle, $400 \mu \mathrm{L} 154$ of $1 \%, 2 \%, 3 \%$, or $4 \%$ NHS- 1 or TM-3 solution was applied 155 to the site of injury (Figure 3, $H$ ).

In another group of anesthetized adult rats the liver was 157 exposed, and a 4-mm punch biopsy done from the ventral 158 aspect through the liver to the dorsal surface of the left liver 159 lobe. The resulting core was removed from the liver, after 160 which one of three treatments was applied. For the treatment 161 group $200 \mu$ L of 3\% NHS-1 solution was applied to the site 162 of injury, whereas in the controls either saline was applied or 163 cauterization of the exposed liver surface was carried out. 164 The superficial material was then wiped clear of the injury 165 site. The abdominal incision was closed, and the animals 166 were allowed to survive for as long as 8 weeks.

\section{Skin punch experiment}

In anesthetized adult nude mice using aseptic precautions, 169 a 4-mm punch was used to create three wounds on each side 170 of the back of the animal. On one side of the animal the 171 wounds created were treated with $1 \%$ NHS- 1 solution, and 172 Q7 the wounds on the opposite side were left untreated to provide 173 a control. The punch biopsies were made through the full 174 thickness of the skin. If the wound did not bleed for 175 10 seconds the punch would be excluded from the data 176 analyzed. All procedures were videotaped, and the analysis 177 consisted of reviewing the tapes. The animals were allowed to 178 survive for as long as 2 months. If animals involved in any of 179 the above experiments appeared to experience any discomfort 180 they were euthanized. 


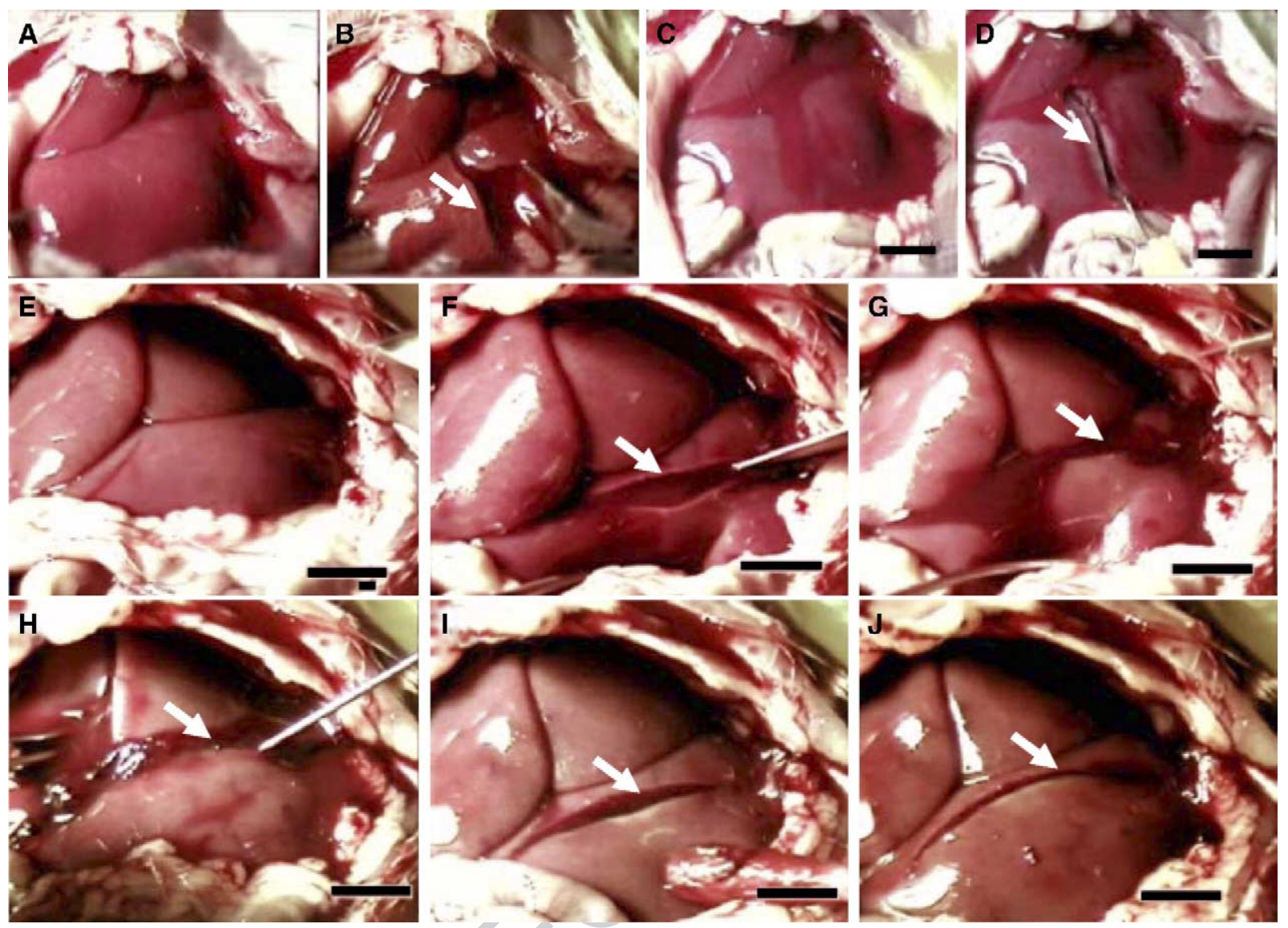

Fig 3. Rat liver hemostasis. This series of pictures is of an adult rat wherein the skin covering the intraperitoneal cavity was excised, exposing the liver. A - D, Sagittal cut. A, The left lateral lobe received a sagittal cut completely transecting a portion of the liver lobe. B, The liver is separated (arrow). Note the profuse bleeding. C, The two halves are allowed to come back together, and the bleeding continues (arrow). D, The 1\% NHS-1 solution was applied, and the extent of the incision was visible under the transparent assembled NHS-1 (arrow). Complete hemostasis was achieved in $8.6 \pm 1.7$ seconds, statistically significant when compared to $90.0 \pm 5.0$ seconds when cauterization was applied, or $301.6 \pm 33.2$ seconds if irrigated with saline. E - J, Transverse cut. This series of pictures is of a transverse cut to the left lateral lobe in an adult rat. E, The exposed intact liver. F, Applying a transverse cut in the lobe (arrow). G, Profuse bleeding produced when a major branch of the portal vein is cut (arrows). H, Treatment with self-assembling NHS-1. Note the complete cessation of bleeding (in $10.3 \pm$ 0.5 seconds using $2 \%$ concentration; $10.0 \pm 1.0$ seconds and $11.0 \pm 1.0$ using $3 \%$ and $4 \%$, respectively) seen under the clear assembled NHS- 1 (arrow). I, 2 minutes after treatment and after the superficial self-assembling NHS-1 has been removed (arrows) to show the extent of cut. J, Bleeding had ceased 15 minutes after NHS-1 treatment. Scale bars represent $1 \mathrm{~mm}$.

\section{Q8182 Transmission electron microscopy sample preparation}

183 In the brain and liver of anesthetized adult rats a $1 \%$ or 184 2\% NHS-1 solution was injected immediately after making $185 \mathrm{a}$ cut, and the treatment site was sampled. Samples were Q9 186 fixed in a mixture of $2 \%$ paraformaldehyde and $2.5 \%$ Q10187glutaraldehyde in $0.1 \mathrm{M}$ phosphate buffer (PB) for 4 hours. 188The samples were washed in $0.1 \mathrm{M} \mathrm{PB}$ three times for 18910 minutes each at $4{ }^{\circ} \mathrm{C}$ and embedded in $2 \%$ agar; blocks Q11190were postfixed in $4^{\circ} \mathrm{C} 1 \%$ osmium tetroxide for 2 hours and 191 then washed in buffer three times for 10 minutes each at $1924^{\circ} \mathrm{C}$. The sample blocks were dehydrated in ethanol, 193 infiltrated, and embedded in pure epon with Lynx EM 194 tissue processor. Ultrathin 70-nm sections were cut (Reich195 ert-Jung ultra cut) and collected on no. 200 mesh grids. 196 Sections and grids were stained with uranyl acetate and lead 197 citrate and examined under a Philip EM208S transmission 198electron microscope.
Preparation of the self-assembling solutions

The NHS-1 solution was prepared using RADA16-I 200 synthetic dry powder (obtained from the Massachusetts 201 Institute of Technology Center for Cancer Research 202 Biopolymers Laboratory, Cambridge, MA; the Zhang 203 laboratory, and 3-DMatrix, Cambridge, MA) dissolved in 204 an Eppendorf tube. The 1\% NHS-1 solution was prepared $205 \mathrm{Q} 12$ by dissolving $10 \mathrm{mg}$ of RADA16-I powder in $1 \mathrm{~mL}$ of 206 autoclaved Milli-Q water (Millipore Corp., Billerica, MA), 207 sonicated for as long as 5 minutes, and filtered. This was 208 repeated with $20 \mathrm{mg} / \mathrm{mL}, 30 \mathrm{mg} / \mathrm{mL}$, and $40 \mathrm{mg} / \mathrm{mL}$ to 209 produce $2 \%, 3 \%$, and $4 \%$ concentrations. NHS-2 and TM-3 $210 \mathrm{Q} 12$ dry powders (made by the Massachusetts Institute of 211 Technology Center for Cancer Research Biopolymers 212 Laboratory, Cambridge, MA) were prepared using the same 213 method. The time of preparation did not affect the action of 214 the solution. We also tested some material that was prepared 215 
A

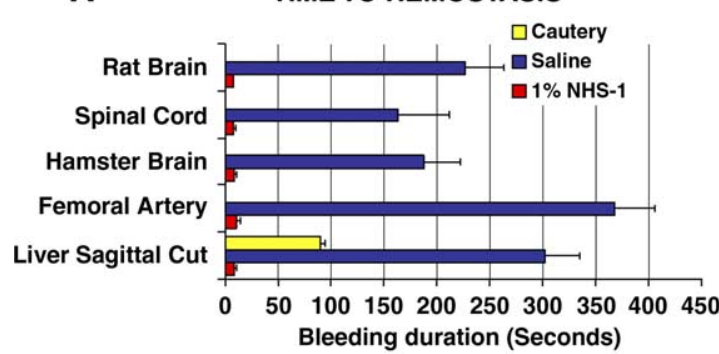

C

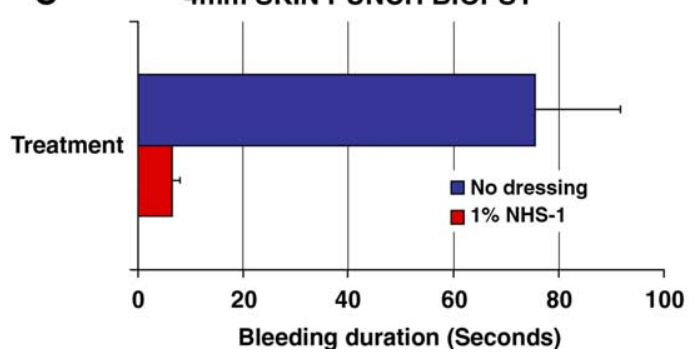

B

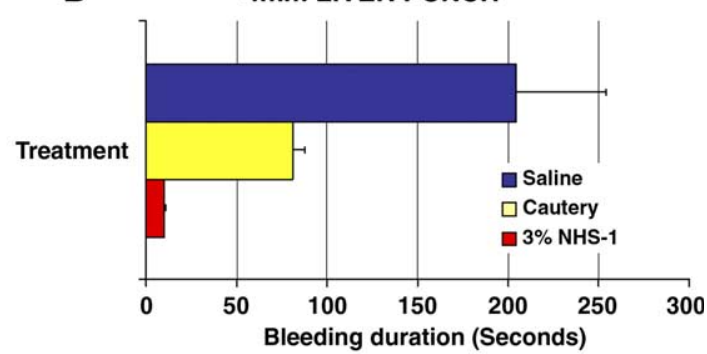

D

TIME TO HEMOSTASIS

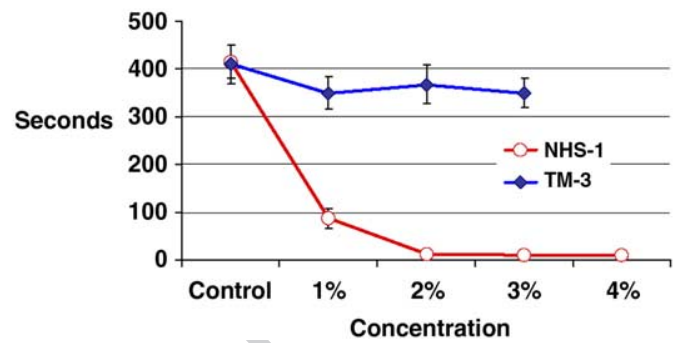

Fig 4. Time required to achieve hemostasis. Graphs illustrate bleeding durations in cases treated with $1 \%$ NHS-1 self-assembling solution compared with those cautery- and saline-treated controls for brain, femoral artery, and liver cuts (A), liver punches (B), and skin punches $(\mathbf{C})$. Each bar shows the mean time in seconds for NHS-1-treated cases (in red), saline controls (in blue), and cautery controls (in yellow). A, In the rat brain cut, durations were measured from the start of application of self-assembling NHS-1 to the completion of hemostasis after transection of the veins leading to the superior sagittal sinus in the brain of adult rats. Complete hemostasis was achieved in $8.4 \pm 2.1$ seconds. In the saline controls bleeding continued until $227.0 \pm 36.6$ seconds. In the hamster brain cut, complete hemostasis was achieved in $9.0 \pm 1.8$ seconds. In the saline controls bleeding continued until $187.6 \pm 34.7$ seconds. In the femoral artery cut, complete hemostasis was achieved in $10.5 \pm 4.1$ seconds. In the saline controls bleeding continued until $367.5 \pm 37.7$ seconds. In the liver sagittal cut, complete hemostasis was achieved in $8.6 \pm 1.7$ seconds. In the cautery control (yellow), bleeding continued until $90.0 \pm 5.0$ seconds, and the saline controls bled for $301.6 \pm 33.2$ seconds. B, Liver 4-mm punch biopsy. A 4-mm core was removed from the left liver lobe, and the hole was treated with NHS-1, heat cautery, or saline. Treatment with 3\% NHS-1 brought about complete hemostasis in $9.7 \pm 1.2$ seconds. In the cautery controls (yellow) bleeding continued for $81.2 \pm 6.7$ seconds, and the saline controls bled for $204.3 \pm 49.6$ seconds. C, Skin 4-mm punch biopsy. A 4-mm punch biopsy was made on the backs of nude mice. The biopsy extended through the dermis, and the core was remoyed. Care was taken not to disrupt the underlying muscle. The three wounds on one side were treated with $1 \%$ NHS-1, and complete hemostasis was achieved in $6.4 \pm 1.5$ seconds. On the opposite side of the animal the wounds were not treated. Bleeding continued until normal clotting occurred at $75.5 \pm 16.3$ seconds. D, Concentration response curves of NHS-1 and TM-3. The left lateral liver lobe received a transverse cut severing a portion of the liver lobe and branch of the portal vein. A higher concentration of NHS-1 (open circles) is more effective in higher pressure and volume hemorrhages. NHS- 1 at concentrations of $4 \%, 3 \%$, and $2 \%$ were effective in achieving hemostasis in $11.0 \pm 1.0$ seconds, $10.0 \pm$ 1.0 seconds, and $10.3 \pm 0.5$ seconds, respectively. The $1 \%$ NHS-1 solution required $86.6 \pm 20.8$ seconds at the area of the most severe bleeding. TM-3 (diamonds) was not effective at any concentration; in the saline controls bleeding continued until $377.5 \pm 85.0$ seconds, and one animal died. Time (seconds) is shown on the $\mathrm{x}$-axis, concentration on the $\mathrm{y}$-axis.

216(obtained from the Zhang laboratory) and stored in solution 217 at room temperature, for 3 years before use, and it 218 performed as well as the newly mixed material.

\section{Results}

\section{Hemostasis in a brain injury}

221 We began our experiments in the brain, removing the 222 overlying skull and performing a complete transection of a 223 branch of the superior sagittal sinus in the brain of rats $(n=$ $22415)$ and hamsters $(n=15)$ (Figure $1, A)$. The areas were 225 treated with $20 \mu \mathrm{L}$ of a $1 \%$ solution of RADA16-I (NHS-1) 226 self-assembling solution or with iced saline. In the groups Q13227treated with NHS-1 hemostasis was achieved in less than 10 228 seconds in both hamsters and rats (Figure 2, $A-D$ and Q14229Supplemental Video 1, "Hemostasis in rat cortex with self230 assembling peptide treatment'). Control group hamsters $231(n=5)$ and rats $(n=5)$ irrigated with saline bled for more 232 than 3 minutes (Figure 4, A). A truncated iced-saline control and subsequent treatment with NHS-1 is shown in 233 Supplemental Video 2 ("Saline control and treatment with 234Q16 self-assembling peptide in rat cortex.") Student's $t$-test for 235 two independent samples in both hamsters and rats showed 236 highly significant differences $(P<.0001)$.

Hemostasis in a spinal cord injury

Because blood has been shown to be toxic in neural tissue 239 [24] we wanted to know if the spinal environment was 240 different from the brain. By quickly bringing bleeding under 241 control secondary damage caused by surgery can be reduced. 242 After laminectomy and removal of the dura, the spinal cord 243 was hemisected at T2, from the dorsal to ventral aspect, 244 and treated $(n=5)$ with $20 \mu 1$ of $1 \%$ NHS-1. Hemostasis $245 \mathrm{Q} 17$ was achieved in just over 10 seconds. In the saline controls 246 $(n=5)$ bleeding continued for as long as 5 minutes. 247 Comparison of the treated group and the saline controls 248 shows a significant difference using the Tukey test with a 249 $99 \%$ confidence interval. 

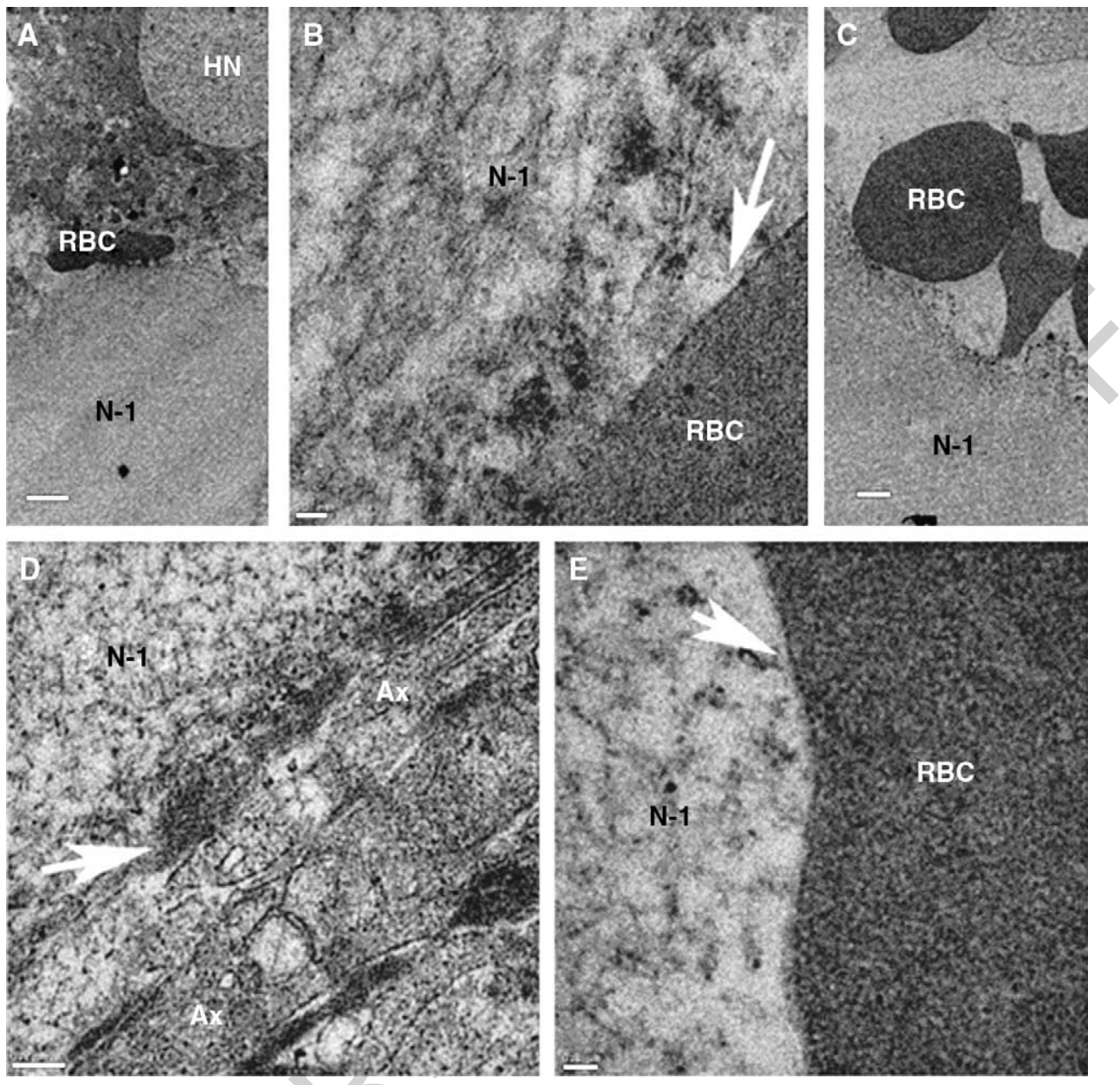

Fig 5. Electron micrographs. This series of TEM images shows the interactions of NHS-1 with liver, cortex, and red blood cells. A, The left lateral lobe was treated with NHS-1, and the tissue was taken shortly after treatment. Note the hepatocyte and its nucleus (HN). There is a red blood cell (RBC) between the assembled NHS-1 (N-1) fields. Scale bar represents $2 \mu \mathrm{m}$. B, A closer look at the interface of the RBC and the material. Scale bar represents $50 \mathrm{~nm}$. C, In the liver the RBC do not appear to mix with the NHS-1. Scale bar represents $1 \mu \mathrm{m}$. D, Application of $1 \%$ NHS- 1 solution to a cut in the cortex. Note the close interface with the axons (Ax). Scale bar represents $0.2 \mu \mathrm{m}$. E, In another part of the brain the interface between the RBC and the NHS-1 appears to be similar to that in the liver. Scale bar represents $0.1 \mu \mathrm{m}$.

\section{Hemostasis in a high-pressure femoral artery wound}

252 The femoral artery of 14 adult rats was surgically exposed, Q17253transacted, and then treated with $200 \mu \mathrm{L}$ of a $1 \%$ solution of 254 NHS-1 or iced saline and packing (Figure 2, E-H). In the 255 treated rats $(n=10)$ about 10 seconds elapsed before 256 hemostasis occurred (Figure $4, A)$. The controls $(n=4)$ 257 continued to bleed for more than 6 minutes. The difference in 258 times to achieve complete hemostasis was highly significant 259(Student's $t$-test $P<.0001$ ).

\section{Hemostasis in highly vascularized liver wounds}

261 Using a group of 76 rats, we performed three different 262 liver cuts: (1) a sagittal (rostrocaudal) cut (Figure 3, $A$ and $263 B$ ) to test NHS-1 in an irregular-shaped laceration wound, 264(2) a transverse (lateral-medial) cut involving the transection 265 of a major branch of the hepatic portal vein to intensify 266bleeding (Figure 3, E-J), and (3) 4-mm punches through the 267 liver lobe to observe the material in uniform wounds.
In the first liver experiment we made a sagittal cut in the 268 left lobe $(n=8)$; upon treatment with $100 \mu \mathrm{L}$ of $1 \%$ NHS-1 269 solution bleeding ceased in less than 10 seconds (Figure 3, 270 $A-D$ and Supplemental Video 3, "Sagittal cut of left liver 271Q18 lobe using $1 \%$ self-assembling peptide treatment"). In one 272 Q19 set of controls $(n=3)$ bleeding stopped 90 seconds 273 (Figure 4, A) after cauterization of the wound; in the 274 saline-treated control animals $(n=3)$ bleeding continued 275 for more than 5 minutes. Comparison of the cauterized and 276 the saline-treated controls shows a significant difference 277 using the Tukey test with a $99 \%$ confidence interval.

278

In the second experiment we severed a major branch of the 279 portal vein while making a transverse cut in the left lobe to 280 test NHS-1 in a environment with a high flow rate. Four 281 concentrations of NHS- 1 were tested $(n=12)$ along with 282 $(n=4)$ control animals. We applied $400 \mu \mathrm{L}$ of $4 \% 283 \mathrm{Q} 19$ concentration NHS-1, and bleeding stopped in 11 seconds 284 (Figure 3, E-J and Supplemental Video 4, "Transverse cut of 28Q20 left liver lobe using $4 \%$ self-assembling peptide treatment"). 286 
Q21287We duplicated the test successfully with $400 \mu \mathrm{L}$ of both $3 \%$ 288 and $2 \%$ NHS-1 solution; bleeding ceased in 10 and 28910.3 seconds, respectively (Figure $4, D$ ). When $400 \mu \mathrm{L}$ of Q212901\% NHS-1 was applied, bleeding continued for more than 29160 seconds (Figure 4, D). The controls, however, bled for 292 more than 6 minutes. The dose response shows that treatment 293 results using $3 \%$ and $4 \%$ NHS- 1 are nearly the same as with 294 the $2 \%$ concentration. Furthermore, in the $2 \%, 3 \%$, and $4 \%$ 295 concentration treatment cases complete hemostasis was 296maintained after removing the excess assembled NHS-1 297 material (Figure 3, $I$ and $J$ ). We found that the higher blood 298 pressure/flow rate transverse liver cut required a concentra299 tion of $2 \%$ NHS-1 or higher to bring about complete 300 hemostasis in less than 15 seconds. A significant difference Q21301was found between the NHS-1-treated and control groups 302 using analysis of variance (ANOVA). When each treatment 303 group was compared to the control group those differences 304 were also significant; a Tukey test showed a 99\% confidence 305 interval. There was no significant difference when the 306 various NHS-1 concentrations were compared, except for 307 the $1 \%$ NHS-1 solution treatment group.

308 In the third experiment using adult rats $(n=45)$ we 309 punched 4-mm holes through the left lateral lobe and then Q21310treated the area with 3\% NHS-1, saline, or heat cautery to 311 bring about hemostasis (Figure 4, B). In the experimental 312 group $(n=15)$ we applied a solution of $3 \%$ NHS-1 after 313 injury and hemostasis was achieved in about 10 seconds, 314 whereas the saline controls $(n=15)$ required 3.5 minutes to 315 stop bleeding. In the heat cautery control group $(n=15)$ 316 cessation of bleeding took more than 60 seconds, inclusive of 317 applying heat to cauterize the inside surface of the punch. We 318 allowed the NHS-1-treated animals to survive for as long as 6 319 months with no detrimental effect on the tissues. Using 320 ANOVA there was a significant difference between the $3 \%$ 321 NHS- 1 treatment and the controls $(P<.0001)$. In addition, 322 the Tukey test showed that each group was significantly 323 different from the other with a $99 \%$ confidence interval.

\section{Hemostasis in skin punch biopsies}

325 Six 4-mm punch biopsies were made on the backs of each 326 of 23 anesthetized adult nude mice for a total of 138 punches. 327 Three punches were treated with $1 \%$ NHS- 1 solution and the 328 other three were left untreated, except for dabbing with cotton 329 every 15 seconds until bleeding stopped. Punched wounds 330 that bled for less than 10 seconds were excluded from the Q21331 experiment. We applied a solution of 1\% NHS-1 10 seconds 332 after injury $(n=23)$, and hemostasis took less than 33310 seconds; the controls $(n=23)$ continued to bleed for 334 more than 60 seconds (Figure 4, $C$ ). The bleeding times were 335 averaged for each side of the animal, and the Student's $t$-test 336 for paired samples showed a significant difference between 337 the treatment and control side of the animal $(P<.0001)$.

\section{Comparison of three different materials}

339 To learn more about the hemostatic properties and 340 mechanism of action of NHS-1 (RADA-16), we repeated both the sagittal and transverse liver experiments, comparing 341 them with two additional materials that are known to self- 342 assemble and spontaneously form nanofibers: (1) RADA-12 343 (NHS-2), a sequence variation of NHS-1, and (2) EAK-16 344 (TM-3), a different sequence in the same family of self- 345 assembling peptides used to determine if the material's 346 length and stiffness altered its hemostatic effectiveness in 347 bleeding models [25-31].

348

Making a sagittal liver cut in adult rats $(n=9)$ we 349 applied $100 \mu \mathrm{L}$ of $2 \%$ NHS-2 solution to the wound, and 350 bleeding stopped in less than 10 seconds. In the cautery 351 controls $(n=3)$ bleeding continued for more than 352 90 seconds $(P<.0001)$. Upon repetition of the experiment 353 in adult rats $(n=8)$ using $100 \mu \mathrm{L}$ of $2 \%$ TM-3, the material 354Q21 assembled but did not achieve hemostasis; the animals 355 continued to bleed until the experiment was terminated after 356 more than 3 minutes.

The increased blood flow from the portal vein after 358 making a transverse liver cut allowed us to perform another 359 dose response experiment in which we compared various 360 concentrations of NHS-1 (1\% to 4\%) and TM-3 (1\% to 3\%) 361 with controls (Figure 4,D). All concentrations of NHS-1 362Q21 were effective; however, the higher blood pressure and flow 363 rate after the transverse liver cut required a concentration of 364 $2 \%$ or higher of NHS-1 to stop bleeding in less than 365 15 seconds.

TM-3 is a stiffer gel; $1 \%$ TM-3 is similar in stiffness to 367 $3 \%$ NHS- 1 . We tried three different concentration levels 368 $(1 \%, 2 \%$, and $3 \%)$ and found that TM-3 was not effective at 369Q21 any concentration; the assembled material fractured and the 370 TM-3-treated animals continued to bleed regardless of the 371 concentration used. There was actually no significant 372 difference between TM-3 and the controls (Figure 4,D) in 373 achieving hemostasis.

Interface of NHS-1 and tissues

375

Still looking for mechanism clues as well as further 376 understanding of the relationship of the NHS-1 blood/tissue 377 interface in both the brain and liver, we also examined the 378 treated tissues using transmission electron microscopy 379 (TEM), interested in learning how the red blood cells (RBCs), 380 platelets, tissue, and the ECM interact with the material. 381

We applied 1\% NHS-1 to a liver wound and immediately 382 harvested the tissue. In the electron micrograph the hepato- 383 cyte and RBC looks to be intact with the assembled NHS-1 at 384 the interface (Figure 5, $A$ ). When applied shortly after injury, 385 the material appeared to stop the movement of blood from the 386 vessels without detrimental effects to the liver's RBCs; there 387 was also no evidence of lysing (Figure 5, B). Furthermore, 388 there was no evidence of platelet aggregation [32] at the 389 blood/NHS-1 interface (Figure 5, C) when samples were 390 taken at various time points after treatment.

In the brain we found a very tight interaction between 392 NHS-1 and the neural tissue (Figure 5, D). We observed no 393 RBCs and no evidence of platelet aggregation in the 394 assembled NHS-1. The RBCs that were present appeared 395 
396 intact at the edges of the assembled NHS-1 with no evidence 397 of lysing (Figure 5, E). Furthermore, no evidence of thrombi 398 was observed in the brain, lung, or liver of the animals 399 treated with NHS-1 and NHS-2.

\section{Discussion}

401 Our study demonstrates that hemostasis can be achieved in 402 less than 15 seconds in multiple tissues as well as a variety of 403 different wounds. This is the first time that nanotechnology 404 has been used to stop bleeding in a surgical setting for animal 405 models and seems to demonstrate a new class of hemostatic 406 agent that does not rely on heat, pressure, platelet activation, 407 adhesion, or desiccation to stop bleeding. NHS-1 and NHS-2 408 are synthetic, biodegradable $[10,19]$ and do not contain any 409 blood products, collagens, or biological contaminants that 410 may be present in human- or animal-derived hemostatic 411 agents such as fibrin glue $[1,8,10,14,20]$. They can be applied 412 directly onto, or into, a wound without the concern that the 413 material may expand, thus reducing the risk of secondary 414 tissue damage as well as the problems caused by constricted 415 blood flow. In our previous brain studies [21] we looked for 416 evidence of the production of prion-like substances or fibril 417 tangles in animals that had the material implanted in their 418 brain for as long as 6 months but found none. Furthermore, 419 the breakdown products of NHS-1 are amino acids, which 420 can be used by the body as tissue building blocks for the 421 repair of the injury [21]. Independent third-party testing of the 422 material found no pyrogenicity, which has been found with 423 some other hemostatic agents, and no systemic coagulation or 424 other safety issues in animals [33].

425 The exact mechanism for the hemostasis reported here 426 is not fully understood, but we have uncovered several 427 clues. First, we know that the hemostasis is not explainable 428 by clotting. Blood clots are produced after injury, but do 429 not begin to form until 1 to 2 minutes have elapsed, de430 pending upon the status and coagulation history of the 431 patient $[6,12,34]$.

432 Second, the electron micrographs show no evidence of 433 platelet aggregation at the interface of the material and wound 434 site. That arginine inhibits platelet aggregation suggests that 435 the arginine in NHS-1 plays a role in this effect [4,35-37]; 436 this seems to be consistent with our data. The NHS-1 and 437 NHS-2 solutions appear to self-assemble into a barrier, 438 stemming the flow of blood and facilitating the movement of 439 adjacent cells to repair the injured site [21].

440 Third, in our experiments the NHS-1 and NHS-2 solutions 441 easily filled in and conformed to the irregular shapes of the 442 wounds before assembling, as shown in the electron micro443 graphs. We believe this tight contact is crucial to the 444 hemostatic action because of the size of the self-assembling 445 peptide units. The micrographs also showed that the material 446 does not cause the RBCs to lyse, apparently protecting them 447 from normal degradation when exposed to the air.

448 Fourth, we do not believe that the hemostasis can be 449 explained by gelation kinetics. One would think that a stiffer gel would be more effective for higher pressure bleeders; 450 however, we found the opposite to be true. TM-3, which is 451 from the same family of peptides as NHS-1 and NHS-2, and 452 is the stiffest of the three self-assembling peptides tested, did 453 not arrest bleeding; it fractured at the tissue interface and 454 within the resultant gel. We surmise that TM-3 may have 455 fractured because of (1) the pulsations of the liver and (2) 456 the inability of the material to flex with the tissue as blood 457 pumped through the organ. This is similar to the fracturing 458 of an artery when grown in a laminar flow environment and 459 then transplanted to a pulsed environment. The cells line up 460 along the direction of flow, unlike the natural helical coil 461 [38-41] seen in a pulsed environment, which allows for 462 expansion and contraction, without splitting, as blood 463 moves though the artery. Conversely, NHS-1 and NHS-2 464 were able to flex with the tissue.

465

Finally, NHS-2, the most pliable of the three materials, 466Q22 seemed to perform identically to NHS-1, probably as a 467 result of their similar structure and modulus.

468

With this discovery the ability to speedily achieve 469 hemostasis will reduce radically the quantity of blood needed 470 during surgery of the future. As much as $50 \%$ of surgical time $471 \mathrm{Q} 23$ can be spent packing wounds to reduce or control bleeding. 472 The NHS solutions may represent a step change in 473 technology and could revolutionize bleeding control during 474 surgery and trauma; however, they still require clinical testing 475 before they can be used in humans.

\section{Acknowledgments}

The authors wish especially to thank both Dr. Ed 478 Tehovnik (Brain and Cognitive Sciences Department, 479 Massachusetts Institute of Technology, Cambridge) and 480 Dr. Chi-Sang Poon (Health Sciences and Technology 481 Department, Massachusetts Institute of Technology, Cam- 482 bridge) for their valuable editing assistance. Thanks also to $48 x 24$ W.W. for assistance in performing the surgary. 484

\section{References}

[1] Alam HB, Burris D, DaCorta JA, Rhee P. Hemorrhage control in the 489 battlefield: role of new hemostatic agents. Mil Med 2005;170:63-9. 490

[2] Bergel G. Über Wirkungen des Fibrins. Dtsch Med Wochenschr 491Q25 $1909 ; 35$.

[3] Blocksom JM, Sugawa C, Tokioka S, Williams M. The Hemoclip: a 493 novel approach to endoscopic therapy for esophageal perforation. Dig 494 Dis Sci 2004;49:1136-8.

495

[4] Dunser MW, et al. Does arginine vasopressin influence the coagula- 496 tion system in advanced vasodilatory shock with severe multiorgan 497 dysfunction syndrome? Anesth Analg 2004;99:201-6.

[5] Grey E. Fibrin as a hemostatic in cerebral surgery. Surg Gynecol 499 Obstet 1915;21:452.

[6] Hambleton J, Leung LL, Levi M. Coagulation: consultative hemo- 501 Q26 stasis. American Society for Hematology Educational Program. 2002. 502 p. $335-52$.

[7] Pallapies D. Vasoactive drugs with an effect on the prostaglandin 504 system. Germ. Wien Klin Wochenschr 1992;104:521-5. 
506 [8] Petersen B, et al. Tissue adhesives and fibrin glues. Gastrointest $507 \quad$ Endosc 2004;60:327-33.

508 [9] Quick AJ. Hemostasis in surgical procedures. Surg Gynecol Obstet $509 \quad 1969 ; 128: 523-32$.

510 [10] Schonauer C, Tessitore E, Barbagallo G, Albanese V, Moraci A. The 511 use of local agents: bone wax, gelatin, collagen, oxidized cellulose. $512 \quad$ Eur J Spine 2004;13(Suppl 1):S89-S96.

513 [11] Kaplan M, Bozkurt S, Kut MS, Kullu S, Demirtas MM. Histopath514 ological effects of ethyl 2-cyanoacrylate tissue adhesive following 515 surgical application: an experimental study. Eur J Cardiothorac Surg $516 \quad 2004 ; 25: 167-72$.

517 [12] De Caterina R, et al. Bleeding time and bleeding: an analysis of the 518 relationship of the bleeding time test with parameters of surgical 519 bleeding. Blood 1994;84:3363-70.

Q27520 [13] Carr Jr ME. Monitoring of hemostasis in combat trauma patients. Mil 521 Med 2004;169:11-5,4.

522 [14] Hoffman M. The cellular basis of traumatic bleeding. Mil Med. 2004; 523 169: 5-7, 4 .

Q28524 [15] Alam HB, Koustova E, Rhee P. Combat casualty care research: from 525 bench to the battlefield. World J Surg 2005.

526 [16] Konturek SJ, Pawlik W. Physiology and pharmacology of prosta527 glandins. Dig Dis Sci 1986;31:6S-19S.

528 [17] Greer IA. Therapeutic progress - review XXVIII. Platelet function and 529 calcium channel blocking agents. J Clin Pharm Ther 1987;12:213 - 22.

530 [18] Kunstlinger F, Brunelle F, Chaumont P, Doyon D. Vascular occlusive 531 agents. AJR Am J Roentgenol 1981;136:151-6.

532 [19] Sabel M, Stummer W. The use of local agents: Surgicel and $533 \quad$ Surgifoam. Eur J Spine 2004;13(Suppl 1):S97-S101.

534 [20] Bhanot S, Alex J. C. Current applications of platelet gels in facial 535 plastic surgery. Facial Plast Surg 2002;18:27-33.

536 [21] Ellis-Behnke RG, et al. Nano neuro knitting: peptide nanofiber 537 scaffold for brain repair and axon regeneration with functional return 538 of vision. Proc Natl Acad Sci U S A 2006;103:5054-9.

539 [22] Wu W. Expression of nitric-oxide synthase (NOS) in injured CNS 540 neurons as shown by NADPH diaphorase histochemistry. Exp Neurol $541 \quad 1993 ; 120: 153-9$.

542 [23] Yick LW, So KF, Cheung PT, Wu WT. Lithium chloride reinforces the 543 regeneration-promoting effect of chondroitinase $\mathrm{ABC}$ on rubrospinal 544 neurons after spinal cord injury. J Neurotrauma 2004;21:932 - 43.

545 [24] Kornecki E, Lenox RH, Hardwick DH, Bergdahl JA, Ehrlich YH. 546 Interactions of the alkyl-ether-phospholipid, platelet activating factor 547 (PAF) with platelets, neural cells, and the psychotropic drugs 548 triazolobenzodiazepines. Adv Exp Med Biol 1987;221:477-88.

549 [25] Zhang S, Holmes T, Lockshin C, Rich A. Spontaneous assembly of a 550 551 598 self-complementary oligopeptide to form a stable macroscopic membrane. Proc Natl Acad Sci U S A 1993;90:3334-8.
[26] Zhang S, et al. Self-complementary oligopeptide matrices support 552 mammalian cell attachment. Biomaterials 1995;16:1385-93.

[27] Holmes TC, et al. Extensive neurite outgrowth and active synapse 554 formation on self-assembling peptide scaffolds. Proc Natl Acad Sci 555 U S A 2000;97:6728-33.

[28] Zhang S, Marini DM, Hwang W, Santoso S. Design of nanostructured 557 biological materials through self-assembly of peptides and proteins. 558 Curr Opin Chem Biol 2002;6:865-71.

[29] Zhang S. Fabrication of novel biomaterials through molecular self- 560 assembly. Nat Biotechnol 2003;21:1171-8.

[30] Caplan MR, Schwartzfarb EM, Zhang S, Kamm RD, Lauffenburger 562 DA. Control of self-assembling oligopeptide matrix formation through 563 systematic variation of amino acid sequence. Biomaterials 564 2002;23:219-27.

[31] Caplan MR, Moore PN, Zhang S, Kamm RD, Lauffenburger DA. Self- 566 assembly of a $\beta$-sheet protein governed by relief of electrostatic 567 repulsion relative to van der Waals attraction. Biomacromolecules 568 2000;1:627-31.

[32] Stassen JM, Arnout J, Deckmyn H. The hemostatic system. Curr Med 570 Chem 2004;11:2245-60.

[33] Zhang S, Zhao X, Spirio L. In: Elisseeff PXMaJ, editor. Scaffolding 572 Q29 in tissue engineering. Boca, Raton, FL: Taylor \& Francis/CRC Press; 573 2005. p. 217-38.

[34] Lind SE. The bleeding time does not predict surgical bleeding. Blood 575 1991;77:2547-52.

576

[35] Cylwik D, Mogielnicki A, Kramkowski K, Stokowski J, Buczko W. 577 Antithrombotic effect of 1-arginine in hypertensive rats. J Physiol 578 Pharmacol 2004;55:563 - 74.

[36] Wang WT, Lin LN, Pan XR, Xu ZJ. Effects of 1-arginine on the func- 580Q30 tion of platelet aggregation during hepatic ischemia/reperfusion in- 581 jury. Chin. Zhongguo Wei Zhong Bing Ji Jiu Yi Xue 2004;16:49 -51. 582

[37] Wang YY, Tang ZY, Dong M, Liu XY, Peng SQ. Inhibition of platelet 583 aggregation by polyaspartoyl 1-arginine and its mechanism. Acta 584 Pharmacol Sin 2004;25:469-73.

[38] Finlay HM, McCullough L, Canham PB. Three-dimensional collagen 586 organization of human brain arteries at different transmural pressures. 587 J Vasc Res 1995;32:301-12.

[39] Sipkema P, van der Linden PJ, Westerhof N, Yin FC. Effect of cyclic 589 axial stretch of rat arteries on endothelial cytoskeletal morphology and 590 vascular reactivity. J Biomech 2003;36:653-9.

[40] Moore Jr JE, et al. A device for subjecting vascular endothelial cells to 592 both fluid shear stress and circumferential cyclic stretch. Ann Biomed 593 Eng 1994;22:416-22.

[41] Ives CL, Eskin SG, McIntire LV. Mechanical effects on endothelial 595 cell morphology: in vitro assessment. In Vitro Cell Dev Biol 1986; 596 22:500-7. 